Faculdade

de Ciências Econômicas UFRGS

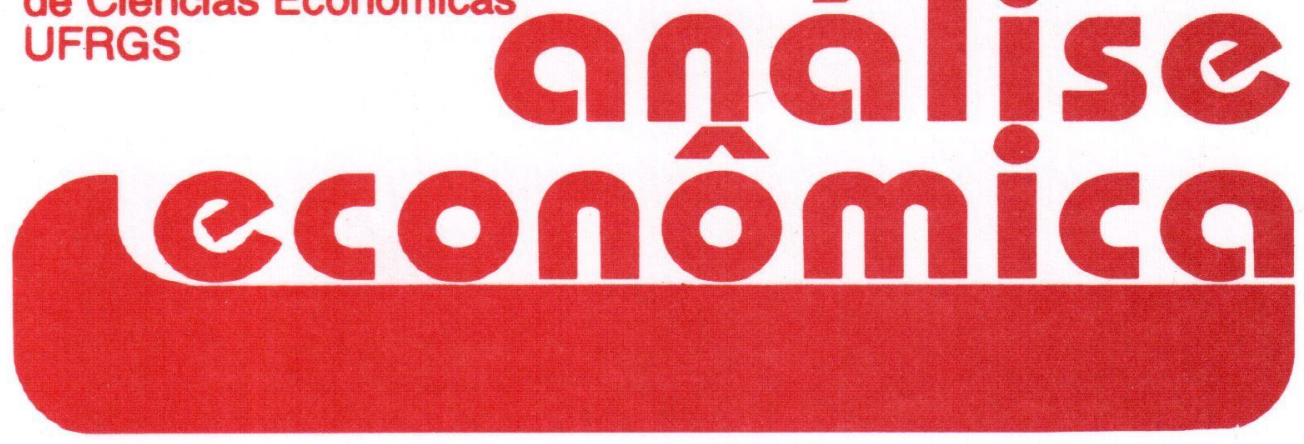

- Finance and Growth:

is Schumpeter Right?

- Philip Arestis

- Panicos Demetriades

- Repensando a

Revolução Marginalista

- Ricardo Luis C. Feijó

- O Papel dos

Rendimentos Crescentes

na Localização Econômica

- Paulo Augusto P. de Britto

- Credibilidade e Mercado Secundário da Dívida Externa Brasileira

- Paulo Calderon

- Rosa Fontes

- Fronteira de Eficiência sob Condições de Risco

- Luís A. de Araújo

- José Vicente Caixeta Filho

- A Criação de

Municípios e seu Impacto na

Qualificação do Espaço Urbano

- Maria Conceição B. Scussel

- Um Estudo Crítico das Relações entre as Poupanças Privada,

Pública e Nacional

- Martin R. Cavalcanti

- Joanílio R. Teixeira
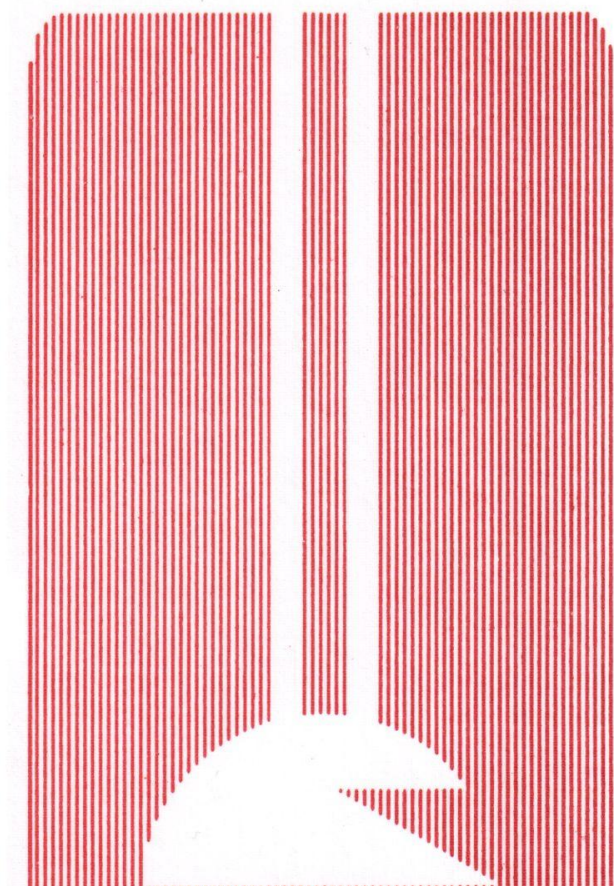

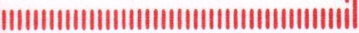

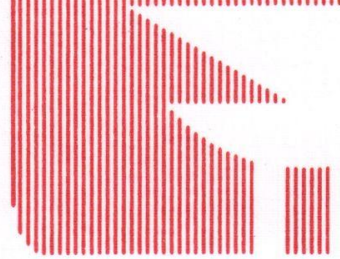

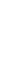


UNIVERSIDADE FEDERAL DO RIO GRANDE DO SUL

Reitora: Prof ${ }^{\mathrm{a}}$. Wrana Maria Panizzi

FACULDADE DE CIÊNCIAS ECONÔMICAS

Diretora: Prof ${ }^{\mathrm{a}}$. Otilia Beatriz Kroeff Carrion

CENTRO DE ESTUDOS E PESQUISAS ECONÔMICAS

Diretor. Prof. Fernando Ferrari Filho

DEPARTAMENTO DE CIÊNCIAS ECONÔMICAS

Chefe: Prof. Luiz Alberto Oliveira Ribeiro de Miranda

CURSO DE PÓS-GRADUAÇÃO EM ECONOMIA

Coordenador. Prof. Marcelo Savino Portugal

CURSO DE PÓS-GRADUAÇÃO EM ECONOMIA RURAL

Coordenador. Prof. Carlos Guilherme A. Mielitz Netto

CONSELHO EDITORIAL: Achyles B. Costa, Aray M. Feldens, Carlos A. Crusius, Carlos G. A. Mielitz Netto, Eduardo A. Maldonado Filho, Eduardo P. Ribeiro, Eugênio Lagemann, Fernando Ferrari Filho, Gentil Corazza, Marcelo S. Portugal, Nali J. Souza, Otília B. K. Carrion, Paulo A. Spohr, Paulo D. Waquil, Pedro C. D. Fonseca, Roberto C. Moraes, Ronald Otto Hillbrecht, Stefano Florissi, Eleutério F. S. Prado (USP), Fernando H. Barbosa (FGV/RJ), Gustavo Franco (PUC/RJ), João R. Sanson (UFSC), Joaquim P. Andrade (UnB), Juan H. Moldau (USP), Paul Davidson (Univ. of Tennessee), Werner Baer (Univ. of Illinois).

COMISSÃO EDITORIAL: Fernando Ferrari Filho, Gentil Corazza, Paulo Dabdab Waquil, Marcelo Savino Portugal, Roberto Camps Moraes.

EDITOR: Eduardo Augusto Maldonado Filho

EDITOR ADJUNTO: Gentil Corazza

SECRETARIA: Revisão de textos: Vanete Ricacheski.

FUNDADOR: Prof. Antônio Carlos Santos Rosa

Os materiais publicados na revista Análise Econômica são da exclusiva responsabilidade dos autores. É permitida a reprodução total ou parcial dos trabalhos, desde que seja citada a fonte. Aceita-se permuta com revistas congêneres. Aceitam-se, também, livros para divulgação, elaboração de resenhas e recensões. Toda correspondência, material para publicação (vide normas na terceira capa), assinaturas e permutas devem ser dirigidos ao seguinte destinatário:

PROF. EDUARDO AUGUSTO MALDONADO FILHO

Revista Análise Econômica - Av. João Pessoa, 52 CEP 90040-000 PORTO ALEGRE - RS, BRASIL

Telefones: (051) 316-3324 e 316-3440 - Fax: (051) 316-3990 rae@vortex.ufrgs.br 


\title{
O PAPEL DOS RENDIMENTOS CRESCENTES NA LOCALIZAÇÃO ECONÔMICA
}

\author{
Paulo Augusto P. de Britto*
}

\section{RESUMO}

A teoria econômica convencional foi construida supondo-se a convexidade das funções de produção, como resultado da presença de rendimentos marginais decrescentes. Isto implica a existência e unicidade do equilíbrio. Atualmente, a existência de retornos crescentes é central nas modernas teorias de desenvolvimento, localização industrial, comércio internacional e geografia econômica. $O$ objetivo deste texto é apresentar quatro modelos que incorporam economias de aglomeração e custos de ajustamento para capturar insights relevantes na determinação da localização industrial e da força de trabalho, e no desenvolvimento econômico. Além disso, busca-se determinar como o approach história versus expectativas determina qual equilibrio de longo prazo é atingido. Os modelos estudados são: Matsuyama (1991), Krugman (1991), Benabou e Fukao (1993) e Arthur (1994).

Cód AEA: 020

Palavras-Chave: Rendimentos crescentes, localização econômica, equilibrio

\section{ABSTRACT}

The classical economic theory was built by supposing convexity of the production function as a result of decreasing returns of scale, which imply the existence and uniqueness of the equilibrium. Actually, the existence of increasing returns is central to the modern theory of development, industrial localization, international trade, and economic geography. The main purpose of this paper is to present four models that use agglomeration economies and adjustment costs to capture important insights. Such that, it is our aim to present how the history vs. expectations works to provide the long run equilibrium. The models studied are: Matsuyama (1991), Krugman (1991), Benabou e Fukao (1993) e Arthur (1994).

AEA Cod: 020

Key Words: Increasing returns, industrial localization, equilibrium

\footnotetext{
"Pesquisador do Centro de Estudos de Empresas da Universidade Católica de Brasília UCB e professor do curso de Pós-Graduação em Gestão Financeira da UCB.

Agradeço os comentários João Ricardo O. Faria (University of Kent e Universidade de Brasília) e de Tatiana Alessio Magalhães (Universidade de Brasília). Como de praxe, os erros e omissões remanescentes são de minha inteira responsabilidade.
}

\begin{tabular}{|l|l|l|l|l|}
\hline ANÁLISE ECONÔMICA & ANO 16 & N.30 & SETEMBRO/98 & p. 47-67 \\
\hline
\end{tabular}




\section{1 - INTRODUÇÃO}

A teoria econômica convencional foi construída supondo a convexidade das funções de produção. Esta convexidade é resultado da presença de rendimentos marginais decrescentes e implica a existência e unicidade do equilíbrio. Este único equilíbrio, chamado equilíbrio de mercado, seria exatamente aquele onde os recursos estariam alocados de maneira mais eficiente. Inversamente, a presença de rendimentos marginais crescentes implica a não garantia de existência e unicidade do equilíbrio e, portanto, instabilidade do resultado final.

A existência de retornos decrescentes atua como força estabilizadora através da minimização dos possíveis efeitos de um choque exógeno. Todavia, em muitos setores da economia moderna, estas forças estabilizadoras não estão presentes. Neste caso, retornos marginais crescentes ampliam efeitos de pequenas variações na economia.

Alfred Marshall, em Principios de Economia (1890), observou que se o custo de produção de uma firma caísse quando sua participação no mercado aumentasse, qualquer firma que, por alguma razão aleatória, obtivesse maior porção inicial do mercado, estaria apta a ser melhor que suas rivais. Marshall não acreditava que retornos crescentes estavam presentes em todos ramos da economia. Agricultura e mineração, as bases da economia de sua época, estariam sujeitas a retornos decrescentes causados por uma limitação na fertilidade da terra ou na qualidade das reservas minerais. Contudo a indústria gozaria de retornos crescentes devido a grandes plantas permitirem melhor organização. Uma conclusão direta a partir de Marshall é que aquelas atividades baseadas em recursos naturais estão sujeitas a retornos decrescentes, enquanto aquelas baseadas em conhecimento estão sujeitas a retornos crescentes.

Atualmente, sabe-se que a não convexidade e os mecanismos de retornos crescentes são centrais nas modernas teorias do comércio internacional, crescimento econômico, organização industrial, macroeconomia, economia regional, desenvolvimento econômico, geografia econômica e política econômica. Os modelos desenvolvidos com retornos crescentes freqüentemente apresentam dois ou mais equilíbrios. A literatura atribui a seleção do equilíbrio do longo prazo à história (conjunto de condições iniciais) ou às expectativas (self-fulfilling prophecy).

Neste trabalho são apresentados três modelos que incorporam rendimentos crescentes para capturar insights relevantes na localização industrial, na localização da força de trabalho e no desenvolvimento econômico. Os modelos apresentados são simples e mostram claramente como o approach história versus expectativas determina qual equilíbrio de longo prazo é atingido. 
No primeiro modelo, Arthur (1994) examinou a dinâmica da localização industrial quando existem economias de aglomeração, ou seja, vantagens de uma firma estabelecer-se numa região onde já existem outras firmas e quando as firmas escolhem sua localização seqüencialmente, sendo a ordem sujeita a acidentes históricos.

No segundo, Krugman (1991) construiu um modelo de um único fator de produção - o trabalho - sujeito a economias externas e custos de ajustamento, a fim de verificar seus efeitos sobre e importância relativa da história e das expectativas na configuração do equilíbrio. Em seguida, apresenta-se a crítica de Benabou e Fukao (1993), que demonstraram estar incorreta a condição terminal utilizada por Krugman (1991).

Em terceiro lugar apresenta-se o modelo de industrialização desenvolvido por Matsuyama (1991). Neste modelo, o autor supõe retornos crescentes no setor manufatureiro e processo de ajustamento em tempo real para analisar em quais circunstâncias a história e as expectativas dos agentes podem determinar ou não o desenvolvimento de uma economia.

Por fim, comparam-se os três modelos, verificando quais as diferenças em sua construção e nas circunstâncias que conferem relevância as condições iniciais e as expectativas na determinação do estado estacionário alcançado a longo prazo.

\section{2 - O MODELO DE ARTHUR}

Arthur (1994) desenvolveu um modelo em que examinou a dinâmica da localização industrial na presença de economias de escala (ou economias de aglomeração) e acidente histórico, supondo que as firmas diferem sensivelmente quanto a suas preferências por certas regiões.

O modelo consiste em $i=1,2, \ldots, n$ firmas, em que cada uma ingressa na economia em um período de tempo. Cada firma difere das demais quanto ao mix de produtos ou quanto à tecnologia empregada na produção. Ao ingressar na economia, cada firma deve optar por se estabelecer em uma dada região, baseando sua escolha no valor presente líquido do ganho de nela estar localizada. Arthur supõe, inicialmente, que o futuro é plenamente descontado, de modo a desprezar as expectativas e assumir que as firmas escolhem a região na qual se estabelecer com base no retorno corrente.

Supondo que cada região proporciona a firma um ganho geográfico ${ }^{1} \mathrm{e}$ que a aglomeração de firmas numa mesma região gera ganhos líquidos, temos:

${ }^{1}$ Exempos de ganho geográfico são: proximidade do mercado consumidor, facilidade na obtenção de insumos e oferta de mão-de-obra qualificada. 


$$
r_{j}^{i}=q_{j}^{i}+g\left(y_{j}\right)
$$

onde $r_{j}^{i}$ é o retorno total da firma i localizar-se na região $j, q_{j}^{i}$ é o ganho geográfico de estar localizado na região $j$ e $g\left(y_{j}\right)$ é o ganho líquido de aglomeração a partir da existência de $y_{i}$ firmas já estabelecidas no momento da escolha.

A diferenciação das firmas quanto ao mix de produtos e à tecnologia empregada na produção gera distinções entre suas preferências geográficas na ausência de outras firmas estabelecidas. As firmas entram na indústria em diferentes instantes e, portanto, fazem sua escolha em seqüência. Arthur introduz a noção de acidente histórico, determinado pela aleatoriedade na seqüência de tipos de firmas que entram. Assim, representa cada nova firma por um vetor $q=\left(q_{1}, q_{2}, \ldots q_{n}\right)$ de preferências locacionais por cada região possível.

A existência de vários equilíbrios torna-se evidente no modelo. Supondo apenas duas regiões, $A$ e $B$, e supondo que as primeiras firmas entrantes optem por se estabelecer na mesma região, digamos $A$, esta passará a apresentar maior ganho de aglomeração sobre as demais reduzindo a importância relativa do ganho geográfico - determinando a concentração da indústria em $A$. De outro modo, se as primeiras firmas entrantes optam por $B$, toda indústria se localizará nesta região. Caso as firmas optem alternadamente por $\mathrm{A}$ e $\mathrm{B}, \mathrm{a}$ indústria será dividida.

Para ver como isto se processa, Arthur tomou a probabilidade de uma firma entrante escolher a região $j$. Supondo existência de $y_{1}, y_{2}, \ldots y_{m}$ firmas já localizadas nas $m$ regiões, a probabilidade da próxima firma preferir se instalar na regiăo $j$ é dada pela probabilidade desta apresentar maior retorno corrente que todas outras regiões individualmente:

$$
\left.p_{j}=\operatorname{prob}\left\{q_{j}+g\left(y_{j}\right)\right]>\left[q_{i}+g\left(y_{i}\right)\right] \forall i \neq j\right\}
$$

Calculando esta probabilidade pela integral de Lebesgue, temos:

$$
p_{j}=\int_{V_{i}} \ldots \int_{V_{j}} \ldots \int_{V_{m}} d F\left(z_{i}, \ldots, z_{j}, \ldots, z_{N}\right)
$$

onde $V_{i}=\left[-\infty, z_{j}+g\left(y_{i}\right)-g\left(y_{i}\right)\right]$ para $i \neq j$ e $V_{j}=(-\infty,+\infty)$.

Assim, o vetor de probabilidade de escolha de uma região, $p=\left(p_{1}, p_{2}, \ldots, p_{N}\right)$, é uma função da dispersão corrente da indústria dada pela função de aglomeração $\mathrm{g}$. 
Representando por $Y_{n}=\left[y_{1}(n), \ldots, y_{N}(n)\right]$ a configuração espacial da indústria no tempo $n$, após $\mathrm{n}$ firmas terem decidido sua localização e por $X_{n}=\left[x_{1}(n), \ldots, x_{N}(n)\right]$ a participação de dada região na indústria ${ }^{2}$, o vetor $p$ de probabilidades de escolha locacional é função de $Y$ ou, equivalentemente, de $X$ e de $n$.

A indústria se forma pela adição de uma, e somente uma, firma em cada período, de modo que o vetor de número de firmas nas regióes potenciais é dado por:

$$
Y_{n+1}=Y_{n}+b\left(n, X_{n}\right) \quad Y(0)=0
$$

onde $b$ é o j-ésimo vetor unitário com probabilidade $p_{j}\left(n, X_{n}\right)$.

Dividindo (4) por $n+1$ e rearranjando, obtém-se a equação que descreve a dinâmica das participações locacionais:

$$
X_{n+1}=X_{n}+\frac{1}{n+1}\left[p\left(n, X_{n}\right)-X_{n}\right]+\frac{1}{n+1} \mu\left(n, X_{n}\right) \quad X_{0}=0
$$

onde $\mu$ é definido como vetor aleatório: $\mu\left(n, X_{n}\right)=b\left(n, X_{n}\right)-p\left(n, X_{n}\right)$.

Calculando a esperança condicionada de (5), podemos descrever o movimento esperado da participação locacional como:

$$
E\left(X_{n+1} / X_{n}\right)-X_{n}=\frac{1}{n+1}\left[p\left(n, X_{n}\right)-X_{n}\right]
$$

Portanto, se a probabilidade $\left(p_{j}\left(X_{n}\right)\right)$ de uma firma entrante se estabelecer na região jé maior que a proporção corrente da indústria na região $j\left(x_{j}(n)\right)$, a participação desta região deve aumentar. Inversamente, se a probabilidade é menor que a proporção corrente, ela deve diminuir.

Após construir a dinâmica do modelo, Arthur passa a fazer considerações sobre as economias de aglomeração.

Supondo a ausência de economias de aglomeração, de modo que $g\left(y_{\mathrm{i}}\right)=0$, somente os aspectos geográficos $q_{j}^{i}$ são considerados. A partir de (3), as probabilidades de escolha $p_{j}$ são constantes e, portanto, independentes da dispersão corrente da indústria. Utilizando-se a lei dos grandes números mostra-se que $x_{j}(n)$ converge para $p_{j}$.

\footnotetext{
${ }^{2}$ Onde $X_{n}=Y_{n} / n$.
} 
Arthur denominou este caso de atração pura, em que a dispersão locacional da indústria é determinada pelo grau de heterogeneidade do tipo das firmas. Se todas firmas são iguais, todas escolherão a mesma região e a indústria se concentrará. Portanto as economias de aglomeração não são condições necessárias para concentração espacial. Então, Arthur propôs o seguinte teorema:

Teorema 1: Quando não existem economias de escala ou aglomeração, ou seja, $g\left(y_{j}\right)=0$, os fatores locacionais são fixos.

Quando as economias de aglomeração estão presentes, a probabilidade de se estabelecer uma nova firma em determinada região depende de adições passadas e, portanto, da história. A partir deste ponto, Arthur analisa dois casos distintos. O primeiro supõe que as economias de aglomeração não são limitadas superiormente. Neste caso, espera-se que o acidente histórico confira a uma dada região uma posição vantajosa (em termos de ganhos líquidos proporcionado à firma entrante) que levará esta a monopolizar toda indústria.

Teorema 2: Quando existem economias de aglomeração ilimitadas, de modo que a função de aglomeração cresce pelo menos à taxa $\partial \mathrm{g} / \partial \mathrm{y}>\varepsilon \mathrm{y}^{\mu}$, onde $\mu>-1$, então a indústria se concentrará toda em uma região. Neste caso, as condições inicias e a seqüência de entrada de firmas desempenham papel relevante na determinação do equilíbrio.

No segundo caso, quando as economias de aglomeração são limitadas superiormente, a indústria não se concentrará necessariamente em apenas uma região. Assim, a indústria poderá se concentrar em um conjunto de regiões exatamente como se as economias de aglomeração estivessem ausentes.

Segundo Arthur, a razão para que isto ocorra, supondo a existência de somente duas regiōes. Considere primeiro que um número suficiente de firmas entre na economia estabelecendo-se na região $A$. Isto confere a esta região uma vantagem, em termos de ganhos líquidos, em relação a $B$, para todas as firmas que futuramente venham a ingressar na economia. Como antes, todas as firmas da indústria se concentrarão na região $A$.

Considere agora que as firmas prefiram $A$ e $B$ mais ou menos na seqüência de entrada. Com isto, estas regiões alcançarão economias de aglomeração equivalentes e nenhuma apresentará vantagem suficiente para eliminar a outra. Sendo estas economias de aglomeração equivalentes, elas deixarão de influenciar na escolha locacional e a indústria se dividirá entre as duas regiões, com quotas que se igualam à proporção dos tipos de firmas que preferem $A$ e $B$ na ausência de economias de aglomeração. 
No segundo caso, o acidente histórico na seqüência de entradas das firmas determina a posição de equilíbrio e, no primeiro, este fator atribui relevância às economias de aglomeração. Portanto a concentração ou a dispersão locacional da indústria terá lugar no modelo de acordo com o acidente histórico na seqüência de entradas.

Teorema 3: Quando houver economias de aglomeração $g\left(y_{j}\right)$ crescentes e limitadas superiormente, a totalidade de indústria se localizará em um grupo de regiões de uma coleção de regiões potenciais. Além disso, as firmas se dividirão entre regiões do grupo que monopolizará a indústria exatamente como se não houvesse economias de aglomeração.

O modelo de Arthur mostra que os argumentos de acidente histórico e economias de aglomeração produzem uma multiplicidade de padrões locacionais possíveis. Mostra que o acidente histórico por si só pode determinar o padrão locacional da indústria e que, contrariamente ao que se poderia esperar, a existência de economias de aglomeração - retornos crescentes na forma espacial - não é suficiente para garantir a concentração industrial em apenas uma região.

Além disso, se as economias de aglomeração apresentam limite superior, mesmo uma pequena heterogeneidade entre as firmas pode causar dispersão de indústria em diversas regiões, exatamente como se estas não existissem. $E$, finalmente, a concentração industrial não é necessariamente resultado da presença de economias de aglomeração, mas da homogeneidade das firmas quanto ao mix de produtos e tecnologias de produção.

\section{3 - O MODELO KRUGMAN}

Krugman construiu uma economia com um único fator de produção, o trabalho, capaz de produzir dois bens distintos. $O$ bem $C$ é produzido com retornos constantes e o bem $X$, com retornos crescentes, ou seja, sujeito a externalidades Marshallianas. Como o preço do fator de produção - salário é igual à produtividade marginal deste, temos que o salário no setor $\mathrm{C}$ é constante e normalizado para 1 . No setor $X$, o salário é dado pela seguinte equação $w=\pi\left(L_{x}\right)$, onde $\pi\left(L_{x}\right)$ é a produtividade marginal do trabalho sujeito a externalidades.

Em virtude da normalização, $w$ é a taxa de salário em $X$ relativa à do setor $C$. Krugman supõe ainda que $\pi(0)<1$ e $\pi(\bar{L})<1$, onde $\bar{L}$ é a oferta total de trabalho da economia. Deste modo, se a taxa de salário no setor $X$ é maior que no setor $C$, todo trabalho será alocado no primeiro e, se a taxa de salário em $X$ for menor que a de $C$, toda força de trabalho será 
empregada no segundo. Evidencia-se assim a existência de múltiplos equilíbrios.

Neste modelo simplificado, as condições iniciais não desempenham papel relevante, pois, na ausência de custos de migração, os trabalhadores se moverão para o setor cuja expectativa de salário é maior, ou seja, para aquele setor cujos agentes esperam que os demais agentes migrem. Assim, na ausência de custos de migração, qualquer equilíbrio pode ser atingido por self-fulfilling prophecys.

Krugman então introduz no modelo custos de ajustamento como função quadrática da taxa na qual os trabalhadores migram entre os setores. Assim, o produto nacional da economia em um dado instante é dado por:

$$
Y=\pi\left(L_{x}\right) L_{x}+\left(\bar{L}-L_{x}\right)-(1 / 2 \gamma)\left(L_{x}^{\&}\right)^{2},
$$

onde $\gamma$ é um parâmetro de velocidade de ajustamento ${ }^{3}$.

Os indivíduos têm por objetivo maximizar o valor presente do produto,

$$
H=\int_{0}^{\infty} Y e^{-r t} d t
$$

Com isto, se estabelece a dinâmica de migração. A taxa, segundo a qual os trabalhadores se movem, será aquela que iguala o custo de migrar para o setor $X$ ao ganho da mudança:

$$
\mathbb{E}_{x}=\gamma q
$$

onde $q$ é o preço sombra de uma unidade de trabalho empregado no setor $X$, definido como:

$$
q(t)=\int_{t}^{\infty}(\pi-1) e^{-r(\tau-t) d \tau}
$$

Diferenciando (9), temos:

$$
r q=(\pi-1)+\&
$$

onde $\pi-1$ é o diferencial corrente de salários entre os dois setores, e \& é a taxa de lucro do capital no ativo sombra. Rearranjando a equação (10) obtemos a dinâmica de $q$ :

$$
\alpha=r q-\pi\left(L_{x}\right)+1
$$

${ }^{3} \mathrm{~A}$ velocidade de ajustamento é o inverso do custo de ajustamento. Portanto, quanto maior for $\gamma$, menor é o custo de ajustamento e vice-versa. $\left(\gamma \in \mathfrak{R}^{+}\right)$ 
As equações (8) e (11) definem um siștema dinâmico no espaço $\left(L_{x}, q\right)$. As leis de movimento estão representadas na figura 1. Facilmente visualizamos que o equilíbrio central é instável, o sistema deve convergir para $E_{c}$, onde a economia se especializa na produções de $C$ ou para $E_{x}$, onde a economia se especializa na produção de $X$.

Neste ponto é necessário estabelecer uma estrutura para o modelo. Krugman supõe uma estrutura linear:

$$
\pi=1+\beta\left(L_{x}-L_{x}^{*}\right)
$$

O sistema composto pelas equações (8) e (11) tem duas raízes, quais sejam: $\rho_{l, 2}=\frac{r \pm \sqrt{r^{2}-4 \beta \gamma}}{2}$; onde ambas são positivas. Deste modo, temos dois casos: um quando $r^{2}>4 \beta \gamma$ e as raízes são reais e determinam as trajetórias em forma de $S$, apresentados na figura 1; e outro quando $r^{2}<4 \beta \gamma$ e as raízes são imaginárias, formando um sistema instável que diverge do centro em expansões oscilatórias, representadas pelas trajetórias em espiral apresentadas na figura 2.

Figura 1

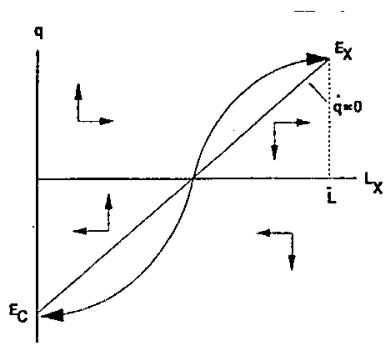

Figura 2

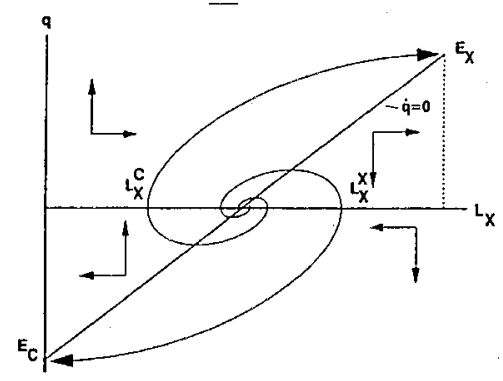

A interpretação econômica da figura 1 diz que a força de trabalho irá se concentrar na região que apresentar mais trabalhadores no período inicial. Portanto, se inicialmente $L_{x}>L_{x}^{*}$ a economia deve se mover gradualmente para $E_{x}$; se inicialmente $L_{x}<L_{x}^{*}$ a economia converge gradualmente para $E_{c}$. Assim, a figura 2 confirma a análise dinâmica ad hoc de que os recursos migram gradualmente para o setor que oferece a maior taxa de salário.

No caso onde as trajetórias são espirais, é importante observar que existe um intervalo determinado pelos braços mais externos destas 
trajetórias. Para todo valor inicial de $L_{x}$ pertencente ao intervalo, qualquer equilíbrio de longo prazo pode ser atingido. Isto é explicado pelas crenças dos agentes, ou seja, se todos os agentes acreditam que os demais trocarão de setor em períodos alternados, farão o mesmo, validando as crenças. Em outras palavras, se a economia parte de uma situação inicial onde, por exemplo, $2 / 3$ dos trabalhadores estão no setor $X$, mas todos esperam que a economia se especialize na produção de $C$, a economia efetivamente se especializará neste último.

O equilíbrio atingido depende das expectativas. Para uma dada posição inicial no intervalo, deve existir um conjunto de expectativas auto-realizáveis que conduzem a algum equilíbrio de longo prazo. Em particular, existem trajetórias definidas pelos braços desenhados no gráfico que conduzem mais rapidamente a alguma posição de longo prazo. Deste modo, o caso das raízes imaginárias é também o caso no qual as expectativas são mais decisivas que a história.

Segundo Krugman, se existe o intervalo determinado pelo overlap e a posição inicial da economia está dentro dele, as self-fulfilling expectations podem conduzir a qualquer equilíbrio. Caso a economia esteja, inicialmente, fora do intervalo, a história determina o resultado final. Se não existe overlap, então história é sempre decisiva neste modelo.

A partir desta constatação, Krugman centrou seu estudo nos fatores que determinam a existência e amplitude do overlap. Estes fatores são a taxa de juros $r$, as economias externas $\beta$ e os custos de ajustamento $1 / \gamma$.

Se a taxa de juros for suficientemente grande, o futuro será fortemente descontado, não importando aos indivíduos as ações futuras dos demais. Se as economias externas não existem, não haverá interdependência entre as decisões. Se a migração é lenta, e o custo de ajustamento elevado, o salário pago em cada setor estará próximo dos níveis correntes por longos períodos de tempo implicando, no limite, que a realocação do fator seja determinada pelos salários correntes. As três condições acima, quais sejam, r elevado, $\beta$ baixo e $1 / \gamma$ elevado determinam a não existência - ou redução

- do overlap e aumento da importância relativa da história.

O modelo de Krugman evidenciou que a importância relativa da história e das expectativas dependem da estrutura subjacente da economia, em particular dos custos de ajustamento.

\section{4 - A CRÍTICA DE BENABOU E FUKAO}

Krugman (1991) construiu um modelo onde a economia apresenta retornos crescentes e economias externas, a fim de verificar seus efeitos 
sobre a importância da história e das expectativas na configuração do equilíbrio. Benabou e Fukao (1993) demonstraram que a condição terminal utilizada por Krugman está incorreta, determinado uma redução no intervalo do overlap e, portanto, na possibilidade de self-fulfilling prophecys.

Partindo do modelo desenvolvido por Krugman, os autores demonstraram que o erro deste foi afirmar que a economia apresenta um dos seguintes pontos terminais: $\quad\left(L_{x}=\bar{L}, q=\{\pi(\bar{L})-1\} / r\right)$ ou $\left(L_{x}=0, q=\{\pi(0)-1\} / r\right)$. Na figura 1 , estes ponto são representados por $E_{X}$ e $E_{C}$, e as trajetórias de equilíbrio de Krugman são representadas pelas curvas sólidas. Matematicamente, Krugman definiu

$$
q(t)=\int_{t}^{\infty}\left(\pi\left(L_{x}(\tau)\right)-1\right) e^{-r(\tau-t)} d \tau
$$

Segundo Benabou e Fukao, as verdadeiras trajetórias de equilíbrio deveriam considerar os pontos terminais $E_{x}^{\prime}\left(L_{x}=\bar{L}, q=0\right)$ ou $E_{C}^{\prime}\left(L_{x}=0, q=0\right)$; estas estão representadas pelas curvas tracejadas na figura 3. Neste caso, a formulação correta de $q(t)$ seria:

$$
q(t)=\int_{t}^{T}\left(\pi\left(L_{x}(\tau)\right)-1\right) e^{-r(\tau-t)} d \tau
$$

onde $T<+\infty$ implica que um dos equilíbrios é alcançado no tempo finito.

Benabou e Fukao argumentaram que o custo de migração quadrático reflete congestionamento, sendo o custo de migrar para cada trabalhador igual a $\left|E_{X}^{\&}\right| / \gamma>0$. Este custo, dado pela equação (8) reflete a quantia que um trabalhador está disposto a pagar para se mover em $t<T$, ao invés de fazê-lo em $T$, quando migrar torna-se menos custoso ${ }^{4}$.

$\mathrm{Da}$ equação (14) podemos concluir que cada trabalhador individualmente tem incentivo em retardar seu movimento até o último instante. Suponha que a economia está localizada no ponto $A$ da figura 3 , sobre a trajetória de equilíbrio de Krugman e na vizinhança do ponto terminal $E_{X}$. Em $A, \mathrm{q}(\mathrm{t})=\frac{\{\pi(\overline{\mathrm{L}})-1\}}{\mathrm{r}}-\varepsilon$ onde $\varepsilon$ é um número positivo muito pequeno. Neste ponto, um trabalhador alocado no setor $C$ pode ganhar ao se desviar do comportamento de equilíbrio. Isto é, ao esperar que todos trabalhadores tenham se movido, ele evita o congestionamento e se move a

${ }^{4}$ Segundo os autores, o último trabalhador a mover teria custo zero. 
custo zero. Com isto, há um ganho de capital $\left|\&_{x}^{\&}\right| / \gamma=q=\{\pi(\bar{L})-1\} / r$ e, em conseqüência, o ponto A não pode ser equilíbrio. A única trajetória que impossibilita este tipo de comportamento arbitrário são as soft landing, quando $\mathrm{q}(\mathrm{t})$ tende a zero. ${ }^{5}$

Benabou e Fukao mostraram então que as verdadeiras trajetórias de equilíbrio são aquelas que conduzem a $\left(L_{x}=\bar{L}, q=0\right)$ ou ( $L_{X}=0, q=0$ ) e que, uma vez alcançado um destes pontos terminais, a economia permanece nele para sempre. Estes pontos terminais são consistentes com o equilíbrio de mercado, uma vez que não restam trabalhadores no setor de menor salário.

Para se derivar a verdadeira trajetória de equilíbrio, deve-se considerar a soma descontada dos salários futuros menos os custos de migração, representados por $v_{x}(t)$ e $v_{c}(t)$. Definindo-se $T$ como o período em que a economia alcança um dos limites $L_{x}=0$ ou $L_{X}=\bar{L}$, e sabendo-se que os trabalhadores optam por permanecer em seu setor até $T, v_{x}(t)$ e $v_{c}(t)$ devem satisfazer:

$$
v_{x}(t)=\int_{t}^{T} \pi\left(L_{x}(\tau)\right) e^{-r(\tau-t)} d \tau+v_{x}\left(T^{-}\right) e^{-r(T-t)}
$$

e

$$
\mathrm{v}_{\mathrm{c}}(\mathrm{t})=\int_{\mathrm{t}}^{\mathrm{T}} \mathrm{e}^{-\mathrm{r}(\tau-\mathrm{t})} \mathrm{d} \tau+\mathrm{v}_{\mathrm{c}}\left(\mathrm{T}^{-}\right) \mathrm{e}^{-\mathrm{r}(\mathrm{T}-\mathrm{t})}, \quad \text { para } \quad \forall \quad t \in[0, T]
$$

onde $v_{i}\left(T^{-}\right)$representa o limite pela esquerda.

Sabe-se que, em qualquer período de tempo $t<T$, os trabalhadores têm a opção de migrar de setor mediante o pagamento do custo de ajustamento $\left|\mathbb{E}_{x}^{\mathbb{E}}\right| / \gamma$. Assim, pode-se determinar as condições necessárias para um trabalhador migrar de $X$ para $C$ e $C$ para $X$, respectivamente:

$$
v_{x}(t) \geq v_{c}(t)-\frac{\left|E_{x}^{\&}(t)\right|}{\gamma}, \quad \text { com igualdade se } E_{x}^{\&}(t)<0
$$

e

\footnotetext{
${ }^{5} \mathrm{~A}$ prova formal das novas condições terminais pode ser vista em Benabou e Fukao (1993), p. 539-541.
} 


$$
v_{c}(t) \geq v_{x}(t)-\frac{\left|\& \varepsilon_{x}(t)\right|}{\gamma}, \quad \text { com igualdade se } E_{x}^{k}(t)>0
$$

Portanto Benabou e Fukao substituíram a definição de Krugman para $q(t)$ pelo seguinte preço sombra, no qual os trabalhadores baseiam suas decisões de movimento, $q(t) \equiv v_{x}(t)-v_{c}(t)$.

Verifica-se que as leis de movimento do sistema permanecem inalteradas. As equações (15) e (16) nos remetem para a equação (11) e as equações (17) e (18), para a equação (8).

A versão de Benabou e Fukao sobre o ponto terminal não invalida as conclusões de Krugman. Ao especificar o mesmo modelo linearizado de Krugman (12), os autores obtiveram as mesmas raízes. Se $r^{2}>4 \beta \gamma$, o sistema diverge a partir de $\left(L_{x}=L_{x}^{*}, q=0\right)$ e a história determinará a trajetória de equilíbrio. Se $r^{2}<4 \beta \gamma$, o sistema diverge em trajetórias expansionistas em espiral e se a posição inicial da economia encontra-se dentro do intervalo determinado pelos braços mais externos do espiral, existirão múltiplas trajetórias determinadas por self-fulfilling expectations.

Finalmente, Benabou e Fukao calcularam explicitamente o overlap do mesmo modo que Krugman. Os resultados são apresentados na Figura 4. A curva sólida plota os valores encontrados por Krugman, enquanto a curva tracejada, os valores de Benabou e Fukao. Assim, o verdadeiro overlap está no intervalo $\left[L_{x}^{c}, L_{x}^{x}\right]$. A redução da amplitude do intervalo implica a menor possibilidade de ocorrência de trajetórias determinadas por self-fulfilling expectations, ou seja, reduz a ocorrência de trajetórias estocásticas determinadas por um dado conjunto de expectativas. A história, e portanto as condições iniciais, tem sua importância relativa ampliada.

Figura 3

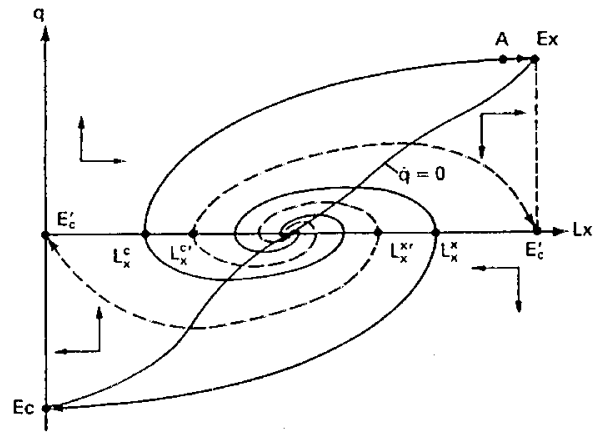

Figura 4

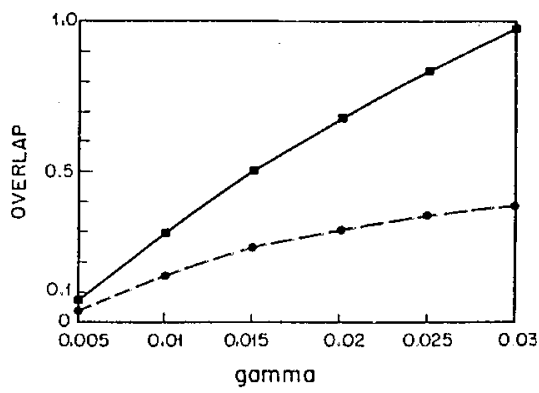




\section{5 - O MODELO DE MATSUYAMA}

Matsuyama (1991) desenvolveu dois modelos de economia, sendo um estático e outro dinâmico, com a presença de retornos crescentes de escala. A existência de economias de escala produz múltiplos estados estacionários. O objetivo principal de Matsuyama é determinar as circunstâncias em que as condições iniciais, ou a história, são relevantes e quando as expectativas são a regra.

\section{1 - Economia estática}

O modelo considera uma economia pequena e, portanto, tomadora de preços. A economia pode ser descrita pelas seguintes suposições sobre tecnologia, agentes e fator de produção.

Tecnologia: Supõe-se que a economia é composta por apenas dois setores e cada setor produz bens homogêneos. O setor agricola opera sob rendimentos constantes de escala, ou seja, $X^{A}=L^{A 6}$. O setor manufatureiro está sujeito a economias de escala externas a firma, o que implica $X^{M}=h\left(L^{M}\right) l^{M}$, onde $l^{M}$ é o emprego da firma. Assim, o função de produção agregada na manufatura é $X^{M}=h\left(L^{M}\right) L^{M}$, onde $h^{\prime}\left(L^{M}\right)>0$.

Agentes: a economia é habitada por um contínuo de agentes, normalizado para 1 . Um agente de tipo $\tau$ pode prover inelasticamente $\mathrm{g}^{\prime}(\tau)$ unidades de trabalho no setor $i$. Um agente $\operatorname{com} \tau$ elevado tem vantagem comparativa na agricultura relativamente a um agente $\operatorname{com} \tau$ baixo. $O$ índice $\tau$ é numerado de forma que $g^{A}(\tau) / g^{M}(\tau)$ é uma função crescente e diferenciável. Seja $T$ a função inversa de $\mathrm{g}^{\mathrm{A}}(\tau) / \mathrm{g}^{\mathrm{M}}(\tau)$, portanto, $\mathrm{T}^{\prime}>0$.

Fator de produção: a economia é dotada de apenas um fator de produção, o trabalho. A taxa de salário no setor agrícola é constante e normalizada para $1, w^{A}=1$. A taxa de salário no setor manufatureiro é determinada segundo a seguinte regra $w^{M}=h\left(L^{M}\right) q$. Considerando o bem agrícola numerário e representando o preço relativo do bem manufaturado por $q$, o salário relativo na manufatura pode ser escrito como

$$
W=h\left(L^{M}\right) q \text {. }
$$

\footnotetext{
${ }^{6}$ Sejam $X^{i}$ e $L^{i}$, respectivamente, o produto e a oferta agregada de trabalho no setor $\mathrm{i}$, medido em unidades eficientes.
} 
Dado um salário relativo $W$, todos agentes cujos tipos são menores que $T(W)$ trabalham na indústria e todos trabalhadores cujos tipos são maiores que $T(W)$ trabalham na agricultura ${ }^{7}$.

Assim, a oferta de trabalho em cada setor é determinada como segue:

$$
L^{A}=Y(W)=\left\{\begin{array}{lc}
\int_{\tau^{-}}^{\tau^{+}} g^{A}(\tau) d \Phi(\tau) & \text { para } T(W) \leq \tau^{-} \\
\int_{\mathcal{T}(W)}^{\tau^{+}} g^{A}(\tau) d \Phi(\tau) & \text { para } \tau^{-} \leq T(W) \leq \tau^{+} \\
0 & \text { para } T(W) \geq \tau^{+}
\end{array}\right.
$$

e

$$
L^{M}=Z(W)= \begin{cases}0 & \operatorname{para} T(W) \leq \tau^{-} \\ \int_{\tau^{-}}^{T(W)} g^{M}(\tau) d \Phi(\tau) & \text { para } \tau^{-} \leq T(W) \leq \tau^{+} \\ \int_{\tau^{-}}^{\tau^{+}} g^{M}(\tau) d \Phi(\tau) & \text { para } T(W) \geq \tau^{+}\end{cases}
$$

onde $L^{A}$ é a oferta de trabalho na agricultura, é a oferta de trabalho na manufatura e $\Phi(\tau)$ é uma função de distribuição de $\tau$ com $\Phi^{\prime}(\tau)>0$ e $\Phi \in\left[\tau^{-}, \tau^{+}\right]$.

O sistema composto pelas curvas de demanda por trabalho (19) e oferta de trabalho (21) na manufatura estão apresentadas na figura 5. A demanda por trabalho na manufatura é positivamente inclinada devido aos retornos crescentes $h^{\prime}\left(L^{M}\right)>0$. A oferta de trabalho também é positivamente inclinada quando $\tau^{-} \leq \mathrm{T}(\mathrm{W}) \leq \tau^{+}$e vertical nos demais casos ${ }^{8}$. Portanto é possível haver múltiplos equilíbrios.

A figura 5 apresenta três equilíbrios: a economia especializada na produção agrícola $S_{0}$, a economia com os dois setores $S_{L}$ e a economia especializada na produção manufatureira $S_{H}$.

${ }^{7}$ Se $\tau^{-}<\mathrm{T}(\mathrm{W})<\tau^{+}$, um salário relativo maior na manufatura atrai mais agentes para este setor. Se $T(W) \geq \tau^{+}$, todos agentes trabalham na manufatura; se $T(W) \leq \tau^{-}$todos agentes trabalham na agricultura.

${ }^{8}$ Quando $T(W)<\tau^{-}$, todos trabalhadores estão empregados na agricultura e quando $T(W)>\tau^{-}$, todos trabalhadores estão empregados na manufatura. 
A seleção do equilíbrio é determinada pelas condições de oferta e demanda por trabalho. Se a posição inicial da economia está entre $S_{O}$ e $S_{L}$, o salário relativo na manufatura é menor que o nível requerido para manter o emprego constante, determinando a fuga de agentes deste setor. A economia converge gradualmente para $S_{0}$. Inversamente, se a economia está inicialmente acima de $S_{H}$, converge para $S_{H}$. Assim, $S_{0}$ e $S_{H}$ são equilíbrio estáveis.

Suponha agora que o emprego inicial na manufatura está entre $S_{0}$ e $S_{H}$. Então o salário relativo na manufatura é maior que o nível requerido para manter o emprego constante, atraindo agentes para este setor e aumentando a oferta de trabalho. Este processo continua até a economia convergir para $S_{H}$. O equilíbrio em $S_{O}$ é instável. A história pode auxiliar na seleção do equilíbrio.

Então, conclui-se que, quando o emprego inicial na manufatura é pequeno, a economia se especializará na produção agrícola, sendo relegada ao subdesenvolvimento. A reversão deste quadro somente seria possivel mediante algum tipo de intervenção governamental. Somente algum instrumento que altere as expectativas poderia tirar a economia da situação determinada pelas condições iniciais.

Matsuyama argumenta que a economia não realiza o salto de $S_{0}$ para $S_{H}$, per si, devido à existência de algum tipo de inércia ou custos de ajustamento que dificultam o movimento instantâneo do trabalho. Mas isto implica que a escolha do setor por um agente é uma decisão de investimento que depende não apenas do salário corrente, mas das expectativas do salário futuro que, devido às externalidades, depende das decisões dos demais agentes.

Surge espaço para as expectativas. O ponto $S_{\mathcal{H}}$ é factível mesmo quando o estado inicial da economia está à esquerda $S_{0}$, isto se todos os agentes acreditam que a economia pode se industrializar e ingressam no setor manufatureiro. Portanto a economia pode vivificar um take off se as expectativas dos agentes forem coordenadas, ou seja, por self-fulfilling optimism. Inversamente, a economia pode desindustrializar-se devido ao self-fulfilling pessimism.

Em suma, a história adquire importância quando os agentes são míopes e descontam completamente o futuro. A questão passa a ser quais as circunstância que concedem relevância à história e quais circunstâncias favorecem as self-fulfilling expectations. 


\section{2 - Economia dinâmica}

O tempo é contínuo com $t \in[0,+\infty[$. A estrutura de produção é idêntica à da economia estática. Supõe a existência de gerações justapostas, mantendo o tamanho total da população constante e igual a 1 ao longo do tempo. Assim, cada agente tem probabilidade instantânea constante $p$ de morrer em cada instante do tempo, o que implica o nascimento de um grupo de pessoas de tamanho $p$.

Supõe que o agente deve escolher em qual setor trabalhar ao nascer e, uma vez tomada a decisão, não pode mais mudar de setor. A irreversibilidade da escolha torna a realocação do trabalho na economia lenta. O suposto da irreversibilidade, adotado por Matsuyama para simplificar o modelo, favorece a história.

Dotado com previsão perfeita, cada agente faz sua escolha objetivando maximizar sua riqueza humana. Portanto o agente escolherá o setor cuja soma descontada dos salários futuros for maior. Isto é, um agente nascido em $t$ irá para agricultura se, e somente se:

$$
\int_{t}^{\infty} g^{A}(\tau) w_{s}^{A} e^{-r(s-t)} d s \geq \int_{t}^{\infty} g^{M}(\tau) w_{s}^{M} e^{-r(s-t)} d s
$$

onde $r>0$ é a taxa de desconto determinada no mercado mundial de capitais. Seja $\theta=r-p$ a taxa da preferência intertemporal. Usando $w_{s}^{A}=1, w_{s}^{M}=h\left(L_{s}^{M}\right) \mathrm{q}$, e a definição de $T$, esta condição pode ser reescrita como $\tau \geq T(Q)$, onde $Q$ é a soma descontada dos salários futuros na manufatura:

$$
Q \equiv r \int_{t}^{\infty} h\left(L_{s}^{M}\right) g e^{-r(s-t)} d s
$$

Assim, a irreversibilidade torna a decisão de um agente dependente também dos salários futuros. Por seu turno, os salários futuros são função do emprego futuro devido às externalidades, e o emprego futuro é função das decisões dos demais agentes.

Pelas definições de $Y$ e $Z$, a oferta agregada de trabalho na agricultura e na manufatura varia de acordo com, respectivamente:

$$
\begin{aligned}
& =P\left[Y\left(Q_{t}\right)-L_{t}^{A}\right] \\
& W_{t}=p\left[Z\left(Q_{t}\right)-L_{t}^{M}\right]
\end{aligned}
$$


onde $Y\left(Q_{t}\right)$ e $Z\left(Q_{t}\right)$ representam o fluxo de entrada de trabalhadores, medidas em unidades eficientes, em cada setor; $L_{t}^{A}$ e $L_{t}^{M}$, o fluxo de retirada de trabalhadores de cada setor devido à morte. Os termos de entrada e saída de trabalhadores são multiplicados por $p$. As dinâmicas de $\left\{\mathcal{L}^{\mathrm{M}}, \mathrm{Q}\right\}$ são independentes de $L^{A}$, de modo que a equação (23) pode ser desprezada.

A dinâmica de $Q_{t}$ é obtida diferenciando-se a equação (22) com respeito ao tempo:

$$
\mathcal{Q}_{t}=r\left[Q_{t}-h\left(L_{t}^{M}\right) q\right]
$$

onde $0 \leq Q_{t} \leq \bar{Q}$ para todo $t \geq 0$.

Portanto, para um dado $L_{0}^{M} \in\left[0, \int_{\tau^{-}}^{\tau^{+}} g^{M}(\tau) \mathrm{d} \Phi(\tau)\right]$, as equações e (25) definem um sistema dinâmico em $\left(L^{M}, Q\right)$ :

$$
\begin{aligned}
& L_{t}=p\left[Z\left(Q_{t}\right)-L_{t}^{M}\right] \\
& \mathcal{Q}_{t}=r\left[Q_{t}-h\left(L_{t}^{M}\right) q\right] .
\end{aligned}
$$

A figura 6 apresenta a dinâmica deste sistema. De (24) o locus $\&=0$ é dado por $Z(Q)=L$. Ele é positivamente inclinado se $\tau^{-}<T(Q)<\tau^{+}$, e vertical nos demais casos. Acima do locus, o emprego na manufatura é crescente e abaixo, decrescente. O locus $\mathcal{Q}=0$ é dado por $Q=h(L) q$, sendo $Q$ crescente acima do locus e decrescente abaixo. 
Figura 5

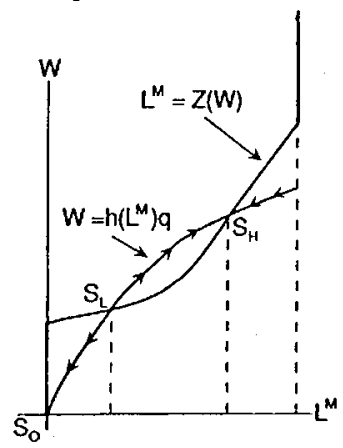

Figura 6

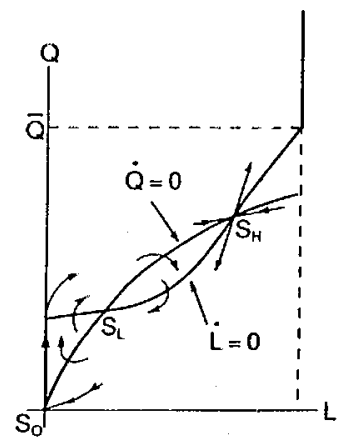

Observe que estes locus são idênticos às curvas de demanda e oferta por trabalho do caso estático. Os estados estacionários desta economia dinâmica coincidem com os equilíbrios da economia estática. A partir de (24) e (25) demonstra-se que $S_{0}$ e $S_{H}$ são pontos de sela; e $S_{0}$ é uma fonte se $\theta>0$ e um poço de $\theta<0$.

A solução do sistema de equações formado por (24) e (25) somente seria possivel se especificássemos uma forma para a função $h$. Esta etapa, realizada por Matsuyama, não será apresentada aqui, mas os resultado foram similares àqueles de Krugman .

Vejamos os principais resultados obtidos. Um importante determinante do papel da história vis-a-vis as expectativas é a taxa de preferência intertemporal $\theta$. Nos casos onde $\theta$ é pequeno as self-fulfilling expectations são favorecidas. Para $\theta$ suficientemente grande, a equação (22) é simplificada de modo que $Q_{t}=h\left(L_{t}^{M}\right) q$, a história sozinha passa a determinar a seleção do estado estacionário, não existindo espaço para as self-fulfilling expectations. Neste caso, a dinâmica é idêntica àquela apresentada na economia estática e, para quaisquer condições iniciais, existe uma única trajetória de equilíbrio com previsão perfeita.

\footnotetext{
${ }^{9}$ A formalização do modelo linear e as soluções são encontradas em Matsuyama (1991) p. 644-646. A análise da dinâmica global também pode ser encontrada no mesmo artigo, p. 629-639.
} 
A intuição é clara, quanto mais descontado for o futuro, menos os agentes considerarão as ações futuras dos demais agentes e mais atenção será dada ao salário corrente, ou seja, às condições iniciais. Outro determinante da importância relativa da história e expectativas são as externalidades. Quanto maior as externalidades, maior a relevância das expectativas e vice-versa.

\section{6 - CONSIDERAÇÕES FINAIS}

Quanto à sua construção, os três modelos apresentam relativa semelhança. Arthur difere um pouco ao estabelecer um modelo probabilístico contra os modelos determinísticos de Krugman-BenabouFukao $^{10}$ e Matsuyama. Além disso, o modelo de Arthur faz referência à diferenciação dos agentes (as firmas), mas a formalização fica por conta de Matsuyama (contínuo de agentes, sendo que cada um apresenta vantagem comparativa no trabalho na indústria ou agricultura). O modelo KrugmanBenabou-Fukao não faz menção alguma sobre diferenciação dos agentes, mas isto fica implícito ao supor que eles migram em instantes diferentes ${ }^{11}$. Nos dois últimos modelos, supôs-se que os agentes apresentam perfect foresight, ou seja, têm conhecimento da existência da tecnologia com retornos crescentes.

Em todos os modelos, a presença de retornos crescentes favorece a história. Em Arthur, estes retornos assumem a forma de economias de aglomeração que começam a operar após as primeiras escolhas, que são determinadas por um acidente histórico na seqüência de entrada. A primeira região preferida concederá economias de aglomeração às novas firmas entrantes e às outras regiões, não.

Nos modelos de Krugman-Benabou-Fukao e Matsuyama, a presença de uma taxa de preferência intertemporal suficientemente grande favorece condições iniciais. A introdução de mecanismos que dificultam ou impedem a locomoção, chamado de custos de migração no primeiro ou o suposto de irreversibilidade do segundo, também favorece a história. Para Matsuyama a irreversibilidade é atribuída a algum tipo de inércia, não necessariamente os custos de migração, mas quaisquer outras razões que dificultam a migração.

Para Krugman a história é sempre determinante num modelo instável (quando as raízes do sistema são reais). No caso do modelo estável (raízes

\footnotetext{
${ }^{10}$ Chamaremos de modelo de Krugman-Benabou-Fukao o modelo de Krugman, incorporadas as correção feitas por Benabou e Fukao.

${ }^{11}$ Enquanto alguns agentes decidem migrar, influenciados pelo diferencial de salário, outros não.
} 
imaginárias), a relevância da história vis-a-vis as expectativas é dada pela estrutura subjacente da economia no que tange, principalmente, às dificuldades de migração (custos), à taxa de juros e à potência das economias externas. Matsuyama analisa, basicamente, a influência de taxa de preferência intertemporal. Quando esta é igual a zero, ou próxima de zero, a história tem papel secundário na seleção do estado estacionário de longo prazo. A coordenação das expectativas podem determinar o estabelecimento de qualquer equilíbrio, seja sob laissez-faire ou intervenção governamental ativa.

A taxa de preferência intertemporal e a taxa de juros determinam o quanto é descontado o futuro. Sendo este fortemente descontado, não há relevância nas decisões futuras dos demais agentes, eliminando a possibilidade de self-fulfilling expectations. Quando a taxa de preferência intertemporal é grande, a história sozinha determina o resultado de longo prazo, não restando papel a ser desempenhado pelas expectativas.

O modelo de Matsuyama no contexto de desenvolvimento econômico é interessante, pois determina por que economias são aprisionadas no subdesenvolvimento e em quais circunstâncias podem fugir deste. A atuação governamental passa a ser considerada na simples promoção do otimismo ou na intervenção ativa.

\section{BIBLIOGRAFIA}

ARTHUR, W. Brain. Increasing Returns and Path Dependence in the Economy. The University of Michigan Press. Ann Arbor, 1994.

BENABOU, Roland e FUKAO, Kyoji. History Versus Expectations: A Comment. The Quarterly Journal of Economics 108(2), May, p. 535-542, 1993.

HENDERSON, J.V. Economic Theory and the Cities. Academic Press, New York, 1977.

KRUGMAN, Paul. History Versus Expectations. The Quarterly Journal of Economics 106(2), p. 651-667, May, 1991.

KRUGMAN, Paul. Geography and Trade. The MIT Pess. Cambridge, 1994.

MARSHALL, Alfred. Principios de Economia. Abril cutural, São Paulo, 1982.

MATSUYAMA, Kiminori. Increasing Returns, Industrialization, and Indeterminacy of Equilibrium, The Quarterly Journal of Economics 106(2), p. 617-650, May, 1991. 